\title{
LINEAR TRANSFORMATIONS THAT PRESERVE THE PERMANENT
}

\author{
PETER BOTTA
}

1. Introduction. Let $M_{m}(F)$ be the vector space of $m$ square matrices over a field $F$. If $X$ belongs to $M_{m}(F)$ we denote by $x_{i j}$ the element in the $i$ th row and $j$ th column of $X$. Let $S_{m}$ be the symmetric group of degree $m$. The permanent of a matrix $X$ in $M_{m}(F)$, denoted by per $X$, is defined by

$$
\text { per } X=\sum_{\sigma \in S_{m}} \prod_{i=1}^{m} x_{i \sigma(i)} .
$$

The permanent is of interest in combinatorial problems [4] and has recently been the subject of several investigations [1], [3]. In a recent paper [1] Marcus and May proved the following:

Theorem. Let $T$ be a linear transformation on $M_{m}(F)$ into itself and suppose per $T(X)=$ per $X$ for all $X$ in $M_{m}(F)$. Then, if $m>2$, there exist permutation matrices $P$ and $Q$ and diagonal matrices $D$ and $L$ such that per $D L=1$ and either $T(X)=D P X Q L$ or $T(X)=D P X^{\prime} Q L$ (here $X^{\prime}$ denotes the transpose of the matrix $\left.X\right)$.

In their proof of this result Marcus and May used the $r$ th permanental compound, a $\left(C_{m, r}\right)$ square matrix analogous to the wellknown $r$ th determinantal compound. The purpose of this note is to prove the above theorem in a somewhat more direct way, avoiding the use of compound matrices. The author would like to thank Professor Marvin Marcus for suggesting this problem to him.

2. Proof of Theorem. In all that follows $T$ is a fixed linear transformation on $M_{m}(F)$ to itself that satisfies per $T(X)=\operatorname{per} X$ for all $X$. We first establish some useful lemmas, some of which may be found in Marcus and May [1].

Lemma 1 [1, Lemma 2]. $T$ is nonsingular.

Suppose $X$ is in $M_{m}(F)$ and $1 \leqq i_{1}<\cdots<i_{t} \leqq m, 1 \leqq j_{1}<\cdots$ $<j_{t} \leqq m$, then by $X\left[i_{1}, \cdots, i_{t} \mid j_{1}, \cdots, j_{t}\right]$ we mean the $t$ square matrix $Y$ defined by $Y_{r s}=X_{i_{r} j_{s}}$ and by $R_{i}\left(R^{j}\right)$ we mean those matrices with row $i$ (column $j$ ) zero.

Lemma 2. Let $A$ belong to $M_{m}(F)$ and suppose $z$ is an indeterminate

Received by the editors July 14, 1966. 
over $F$. If for all $X$ in $M_{m}(F)$, deg per $(X+z A) \leqq 1$ then for all $i<j$, $k<l$ per $A[i, j \mid k, l]=0$.

Proof. A straightforward computation shows that the coefficient of $z^{2}$ in the polynomial per $(X+z A)$ is

$$
\sum_{\sigma \in S_{m}}\left(\sum_{i<j} a_{i \sigma(i)} a_{j \sigma(j)} \prod_{t=1 ; t \neq i, j}^{m} x_{t \sigma(t)}\right) .
$$

For fixed $i<j$ let $\sigma$ in $S_{m}$ be the permutation interchanging $i$ and $j$. Choose $B$ in $M_{m}(F)$ such that $b_{t t}=1$ for $t \neq i, j$ and $b_{r s}=0$ otherwise. Then the coefficient of $z^{2}$ in $\operatorname{per}(B+z A)$ is $\operatorname{per} A[i j \mid i j]$ which by assumption is zero. The result now follows if we observe that for any two permutation matrices $P$ and $Q$, per $P A Q=\operatorname{per} A$.

A result similar to the above has been used [2] in considering a similar problem for the determinant function.

Lemma 3 [1]. Suppose $A$ is in $M_{m}(F)$. Then per $A[i, j \mid k, l]=0$ for all $i<j, k<l$ if and only if either

(1) $A$ belongs to $R_{i}\left(R^{i}\right)$ for some $i$.

(2) There exist $r<s, u<v$ such that

$$
A[u, v \mid r, s]=\left(\begin{array}{cc}
x & -z x / y \\
y & z
\end{array}\right)
$$

for some $x, y, z \neq 0$ in $F$ and all other entries in $A$ are zero.

Sketch of Proof. Clearly if $A$ is of either form then per $A[i, j \mid k, l]$ $=0$ for all $i<j, k<l$. Suppose $A$ is not of either form and all 2 square subpermanents of $A$ vanish. Then there exist $i<j, k<l$ such that $a_{i k} \neq 0, a_{j l} \neq 0$ and $(\alpha, \beta)$ different from any of $(i, k),(i, l),(j, k)$, $(j, l)$ such that $a_{\alpha \beta} \neq 0$. If we consider the submatrices $A[i, j \mid k, l]$, $A[\alpha, i \mid \beta, l]$ and $A[\alpha, j \mid \beta, k]$ and use the fact that the permanents of these must vanish we soon arrive at a contradiction.

We now turn to the proof of the theorem. Let $z$ be an indeterminate over $F$. If $A \in R_{i}\left(R^{j}\right)$ then, by Lemma $2, \operatorname{deg}$ per $(X+z A) \leqq 1$ for all $X$. Therefore deg per $(T(X)+z T(A)) \leqq 1$ for all $X$. By Lemma $1, T$ is nonsingular hence as $X$ runs over $M_{m}(F)$ so does $T(X)$. It now follows that $T(A)$ is of one of the forms described in Lemma 3. We show that the second form cannot occur. To this end it is convenient to consider the entries of $T(A)$ as linear forms in the entries of $A$. Let $z_{1}, \cdots, z_{m}$ be indeterminates over $F$. We let $E_{i j}$ be the matrix with a 1 in the $(i, j)$ position and zeros elsewhere

$$
Z=\sum_{t=1}^{m} z_{t} E_{i t}
$$




$$
(T(Z))_{r s}=L_{r s}\left(z_{1}, \cdots, z_{m}\right) .
$$

It follows that $T(Z)$ must have one of the forms described in Lemma 3 for if not, we could choose $z_{t}=a_{t} \in F$ such that

$$
A=\sum_{t=1}^{m} a_{t} E_{i t} \in R_{i}
$$

and $T(A)$ would not have the required form. Suppose $T(Z)$ has the second form. Then for some $i<j, k<l$ we have

$$
T(Z)[i, j \mid k, l]=\left(\begin{array}{ll}
L_{i k}\left(z_{1}, \cdots, z_{m}\right) & L_{i l}\left(z_{1}, \cdots, z_{m}\right) \\
L_{j k}\left(z_{1}, \cdots, z_{m}\right) & L_{j l}\left(z_{1}, \cdots, z_{m}\right)
\end{array}\right)
$$

and all other elements of $T(Z)$ are zero. Further, since deg per ( $Y$ $+z T(Z)) \leqq 1$ for all $Y$ (as a function of $z$ ), all 2 square subpermanents of $T(Z)$ must vanish. This observation, in conjunction with the unique factorization theorem for polynomials in several variables, shows that either

$$
L_{i k}\left(z_{1}, \cdots, z_{m}\right)=L_{i l}\left(z_{1}, \cdots, z_{m}\right)
$$

and

$$
L_{j k}\left(z_{1}, \cdots, z_{m}\right)=-L_{j l}\left(z_{1}, \cdots, z_{m}\right)
$$

or

$$
L_{i k}\left(z_{1}, \cdots, z_{m}\right)=-L_{j k}\left(z_{1}, \cdots, z_{m}\right)
$$

and

$$
L_{i l}\left(z_{1}, \cdots, z_{m}\right)=L_{j l}\left(z_{1}, \cdots, z_{m}\right)
$$

to within a permutation of rows and columns.

The two linear forms $L_{i k}$ and $L_{j l}$ must involve all of the indeterminates because $T$ is nonsingular. We have assumed $m \geqq 3$ so we can choose $a_{1}, \cdots, a_{m}$ in $F$ not all zero such that $L_{i k}\left(a_{1}, \cdots, a_{m}\right)$ $=L_{j l}\left(a_{1}, \cdots, a_{m}\right)=0$. This however implies that $T$ is singular, a contradiction. It now follows that $T\left(R_{i}\right)=R_{j}$ or $R^{j}$ for some $j$ and that $T\left(R^{t}\right)=R_{k}$ or $R^{k}$ for some $k$. If we recall that $T$ is nonsingular and onto then it is not hard to check that either

(i) $T\left(R_{i}\right)=R_{i}^{i_{i}}$ and $T\left(R^{i}\right)=R_{k_{t}}$ or

(ii) $T\left(R_{i}\right)=R_{j_{i}}$ and $T\left(R^{t}\right)=R^{k t}$

for all $i, t$ and that $j_{i}=\sigma(i), k_{t}=\tau(t)$ for suitable permutations $\sigma, \tau \in S_{m}$. Suppose the first case occurs. Then

$$
T\left(E_{i j}\right)=\sum_{s=1}^{m} c_{s} E_{s \sigma(i)}=\sum_{r=1}^{m} d_{r} E_{\tau(j) r}
$$


Now $(s, \sigma(i))=(\tau(j), r)$ if and only if $s=\tau(j), \sigma(i)=r$. The matrices $E_{k l}, k, l=1, \cdots, m$ are linearly independent so it follows that $T\left(E_{i j}\right)$ $b_{i j} E_{\tau(j) \sigma(i)}$ for some $b_{i j} \in F$.

Let $P, Q$ and $B$ be defined by

$$
P_{i j}=\delta_{i \sigma^{-1}(j)}, \quad Q_{i j}=\delta_{i \tau(j)}, \quad B_{i j}=b_{i j} .
$$

Then a straightforward computation shows that

$$
T(X)=B *\left(P X^{\prime} Q\right)
$$

where ${ }^{*}$ is the Hadamard product defined by $\left(R^{*} S\right)_{i j}=R_{i j} S_{i j}$, and $\delta_{r s}=1$ if $r=s$ and 0 otherwise.

If the second case occurs we may show in a similar way that $T(X)$ $=B *(P X Q)$ for permutation matrices $P$ and $Q$ and a suitable matrix $B$.

By choosing $X$ to be an appropriate permutation matrix we find that for each $\omega \in S_{m}$

$$
\prod_{i=1}^{m} b_{i \omega(i)}=1 ;
$$

so for any $i, j, \quad b_{i j} \neq 0$. Hence by choosing $\omega$ and $\mu$ to be appropriate transpositions we have $b_{i r} b_{j 8}=b_{i s} b_{j r}$ for all $i, j, r, s$. Set $a_{i}=b_{i 1} / b_{11}$; $c_{j}=b_{1 j}$ then $a_{i} c_{j}=b_{i 1} b_{1 j} / b_{11}=b_{i j}$. Hence if $A=\operatorname{diag}\left(a_{1}, \cdots, a_{m}\right)$; $C=\operatorname{diag}\left(c_{1}, \cdots, c_{m}\right)$ we have that for any $X \in M_{m}(F)$

$$
B * X=A X C \text {. }
$$

Also per $A C=\prod_{i=1}^{m} a_{i} c_{i}=\prod_{i=1}^{m} b_{i i}=1$.

This completes the proof of the Theorem.

\section{REFERENCES}

1. M. Marcus and F. May, The permanent function, Canad. J. Math. 14 (1962), 177-189.

2. M. Marcus, Linear operations on matrices, Amer. Math. Monthly 69 (1962), 837-847.

3. M. Marcus and H. Minc, On the relation between the permanent and the determinant, Illinois J. Math. 5 (1961), 376-381.

4. H. J. Ryser, Compound and induced matrices in combinatorial analysis, Proc. Sympos Appl. Math., Vol. 10, pp. 149-167, Amer. Math. Soc., Providence, R. I., 1960.

UNIVERSITY OF MichigAN 